\title{
Standard of Care in Clinical Research Involving Human Subjects: A Perspective from Developing World
}

\author{
Muhammad Waseem Khan ${ }^{1}$, Sanam Zeib Khan ${ }^{2}$, Afrasiab Khan Tareen ${ }^{3}$, Imrana Niaz Sultan ${ }^{4}$ \\ 1,2,3,4, Department of Biotechnology \& Informatics, Faculty of Life Sciences \& Informatics, Balochistan \\ University of Information Technology Engineering \& Management Sciences (BUITEMS) Quetta \\ Balochistan, Pakistan.
}

Email: muhammad.waseem@buitms.edu.pk, mwaseem.tareen@yahoo.com

\begin{abstract}
Standard of care is an ordinary, reasonable formal treatment and diagnostic process that a physician should follow for his/her patient with specific disease. Standard of care followed in one country may not suit to other country; the reason may involve economic conditions, certain norms, beliefs, tradition and culture of that society. It may be considered ethical if it does not exploit human rights and poor vulnerable population, once it exploits the human rights it creates ethical dilemmas that need to be sort out to protect vulnerable population and to make the research more ethical. Patients attending medicine department of Bolan Medical Complex (BMC) Quetta, Balochistan, Pakistan were selected randomly interviewed and requested to fill the questionnaire. The ethical issues in clinical research conducted on human population have been perplexing and remains to be the same in clinical research settings. Exploitive use of research participants in resource poor developing countries has intensified the debate on the ethics of international research and led to increasing attention to exploitation of vulnerable study subjects. The issues of conducting research in developing countries will remain and need to be focused and debated where and whenever require. One has to try sincerely to sort out the ethical problems while conducting a research study, he might fail to solve all the issues but the situation may improve by the time if tried sincerely.
\end{abstract}

Keywords: Global Standard, Placebo, Resource Allocation, Clinical Research, Post-trial Benefits

Introduction: Standard of care is defined as an ordinary, reasonable formal treatment and diagnostic process that a physician should follow for a patient with certain and specific symptoms or disease. Standard of care is the attention and prudence that a reasonable researcher and physician in the circumstances would try to administer in practice. If a person's actions do not meet this standard of care, then his/her acts fail to meet the duty of care which all people (supposedly) have toward others. Failure to meet the standard of care is termed as negligence, and any damages resulting there from may be claimed in a lawsuit by the injured party. A standard of care may differ from one community to the other. Standard of care followed in one country may not suit to other country; the reason may involve economic conditions, certain norms, beliefs, tradition and culture of that society. It may be considered ethical if it 
does not exploit human rights and poor vulnerable population, once it exploits the human rights it creates ethical dilemmas that need to be sort out to protect vulnerable population and to make the research more ethical.

In developing countries ethics play a major role in health care and in health research, as better health of any community is the main focus of any government whether in development or developed country for its benefit and development. The recent debate in developing countries is mainly focused on the issue of standard of care that is used for the research participants in research studies that are conducted by multinational companies from developed countries. There are other issues such as voluntary inform consent, community participation, risk benefit ratio, fair subject selection, post trial benefits and many more to be taken care of. The important of all is the issue of autonomy, beneficence and justice that need to be focused and should be considered as primary responsibility of any researcher conducting any research study, it almost covers all other issues that may be labeled as the secondary issues ${ }^{1}$.

The ethics of research on human population has been a challenging and remains to be the issue of great debate. Exploitive use of research participants in resource poor developing countries has intensified the debate on the ethics of international research and led to increasing attention to exploitation of vulnerable study subjects. As only less than $10 \%$ of the world research resources are allocated for the $90 \%$ of health problems that causes the debate of standard of care which should be provided to the study participants in research studies that are conducted in developing countries ${ }^{1}$.

Declaration of Helsinki 1996 version answers it, that worldwide best possible available proven standard of care should be provided, but it has been widely rejected by national as well as multinational committees worldwide. Interestingly it is interpreted as the best available treatment available in the country where the research is conducted and due to this interpretation, that research study is considered ethically permissible which provide less than possible available standard of care in some circumstances, if not in all situations ${ }^{2}$.

There is more than one possibility when standard of care is set which have not been clearly distinguished from one and other. In the presence of these possibilities it becomes difficult to assess the implications of opposing or accepting certain type of standard of care in any research study. The declaration of Helsinki which states "best proven therapeutic method" it is interpreted as the best therapy available anywhere in the world it is given the name as "Global reference point" (Global defacto) or the therapy or the standard of care available and used in the country where the research study is conducted, it is named as "Local reference point" (Local defacto). 
Following global reference point may be deemed as most suitable for the people of developing countries where the study is conducted, but it is considered as burden by the trial conducting companies or the sponsoring countries. The local reference point make life easy for the researcher to use placebo or no treatment where there is no treatment at all in that country therefore, the use of a placebo in control group is not considered as unethical on the ground that it fails to provide the established standard of care as the standard of care is considered of that country. The use of a placebo in the subjects of control group in these countries does not fall below the established standard of care of that country. While considering the global reference point will not allow the use of placebo or low standard drug in control arm as the researcher has to follow the global standard point that does not take care of the standard of care that is prevailing in the research conducted country ${ }^{3}$.

It is argued that if the researchers are obtaining some useful data for the developing countries, some might get less than best standard of care which balances the weight, but all in all it is unethical to do so and it also violates the mutual relationship of trust that research participant has on the researcher. The Declaration of Helsinki rejects the use of placebo in trials which causes irreversible harm to the research participant and the use of placebo might be considered ethical and less harmful in the trials of less severe diseases, which do not cause irreversible harm. ${ }^{2}$ Secondly, the use of placebo is considered in trials where no proven diagnostic or therapeutic treatment is available, where no treatment might also be considered. In these settings it is deemed as unethical to conduct a clinical trial in which some subjects receive a level of care that falls below the established standard of care ${ }^{2,4}$.

To avoid the exploitation of host communities where research is conducted, subjects must receive the best methods available worldwide on the other hand this strict requirement may block important research intended to improve health care, especially in developing countries that are already conducted for the benefit of the poor communities or at least they are getting some sorts of benefits from that ${ }^{5}$.

Standards of care that are applied for the research conducted in the developing countries should be the same as those applied in developed countries. A single moral standard should govern all research on human subjects, regardless of where and when the research is carried out rather than applying different for developing countries than developed countries. It may be argued that sticking strictly for global standard principle might stop a significant amount of important research which is taken out in developing countries. In such cases, after satisfying the issues of autonomy, justice and beneficence the standard of care might be considered as what is usually available in a developing country i.e. in country where the research study is conducted. In cases where research is conducted in that country where there is total absence of care or health services or there is no treatment for the disease about which the research study is conducted, the use of placebo or no treatment in control arm should not be considered as a suitable control standard, and in these situations the use of placebo in control arm should not be allowed 
but instead, in these cases best available treatment i.e. global standard of care should be used for the subjects in control arm. After all the research conducting companies are taking benefits out of the research study that is being conducted in developing countries ${ }^{4}$.

It may dissatisfied with a standard of care to define it only in relation to the treatment or drug that is being provided to the control arm of any research clinical trial, but it should be considered in a broader sense keeping in mind the most important issue that is the ethics of research in developing countries. Problems dealing with ethical issues in research if given proper importance would solve all other issues regarding standard of care in developing countries or anywhere in the globe.

Collaborative and mutual partnership between the researchers and the study participants that starts from proper informed consent following the principles of autonomy, justice and self respect, determining health problems, respecting community's values, norms, traditions and culture helps to sort out ethical dilemmas. Properly assessing the value of research being conducted that specifies the beneficiaries play a major role in avoiding the subject exploitation.

In any research study the vulnerable population should be protected at all costs and the study participants should be fairly selected without any biasness. Favorable risk-benefit ratio that determines the risks associated with conducting the trial should be clearly mentioned and the study participants should be given more benefit than the associated risks ${ }^{6}$.

Another important issue is the post trial benefits that should be focused and given importance especially once the research is over, pointing out that the new intervention proves to be effective and functioning then it should be offered to those in the control arm of the study subjects once the research is over ${ }^{4}$. 
Conclusion: Following four basic ethical principles of Autonomy, Justice, Beneficence and Nonmaleficence might help to reduce the ethical issues while conducting any clinical trial in resource poor settings, but the issues of conducting research in developing countries will remain a worry and need to be focused and debated where and whenever required. One has to try sincerely to sort out the ethical problems while conducting a research study. Researchers following ethical rules might fail to solve all the issues but the situation may improve by the time if tried sincerely.

\section{References}

1. Bhutta, Zulfiqar Ahmed (2002). Ethics in international health research: a perspective from the developing world. Bulletin of the World Health Organization; 80:114-120.

2. R K Lie, E Emanuel, C Grady, et al (2004). The standard of care debate: the Declaration of Helsinki versus the international consensus opinion. J Med Ethics 30: 190-193.

3. London, Alex John (2000). The Ambiguity and the Exigency: Clarifying 'Standard of Care' Arguments in International Research', JM\&P, 25: 4, 379 -397.

4. J R McMillan and C Conlon (2002). The ethics of research related to health care in developing countries. J Med Ethics, 30, 204-206.

5. Wendler et al (2004). The Standard of Care Debate: Can Research in Developing Countries Be Both Ethical and Responsive to Those Countries' Health Needs? Health Policy and Ethics Forum, American Journal of Public Health, 94:6.

6. Emanuel et al (2004). What Makes Clinical Research in Developing Countries Ethical? The Benchmarks of Ethical Research, 20040: 189.

Competing interests: The authors declare that they have no competing interests.

Acknowledgements: The authors would like to acknowledge the generous support and guidance of Dr. Arshi Farooqui, Dr. Maria Zawyar and Dr. Arsalan Khan.

Authors' contributions: All authors have contributed substantially to the conception and design of the manuscript. Muhammad Waseem Khan is the $1^{\text {st }}$ and corresponding author. Sanam Zeib Khan, Afrasiab Khan Tareen, and Imrana Niaz Sultan are $2^{\text {nd }}$ authors and they have contributed equally. Muhammad Waseem Khan has critically revised the manuscript. All authors have read and approved the final manuscript.

Funding: The Authors declare that the current study was not funded. 